\title{
Fault-Tolerance in Cyber-Physical Systems: Literature Review and Challenges
}

\author{
Luis Piardi*†, Paulo Leitão*, André Schneider de Oliveira ${ }^{\dagger}$ \\ * Research Center in Digitalization and Intelligent Robotics (CeDRI), Instituto Politécnico de Bragança, \\ Campus de Santa Apolónia, 5300-253 Bragança, Portugal \\ Email: \{piardi, pleitao\}@ipb.pt \\ $\dagger$ Graduate School of Electrical Engineering and Computer Science (CPGEI), \\ Universidade Tecnológica Federal do Paraná (UTFPR), \\ Avenida 7 de Setembro 3165, Curitiba 80230-901, Paraná, Brazil \\ Email: andreoliveira@utfpr.edu.br
}

\begin{abstract}
Modern industry demands techniques that ensure the operability of its processes, and even though the exponential technological advance in the last two decades in the manufacturing field, failures, waste, and unexpected interruptions are still present in this sector's daily routine. Within the Industry 4.0 context, fault-tolerant (FT) production systems remain a complex issue and sometimes represent a vulnerable aspect. Faulttolerance techniques dedicated to autonomous and distributed systems, in a cyber-physical system (CPS) perspective, need to be investigated to follow the evolutionary pace of the manufacturing scenarios. This paper overviews these concepts and analyses the current situation in developing FT for CPS systems through a systematic literature review. The paper also discusses the research challenges in this new kind of FT systems due to new distributed architectures and emerging technologies, matching the several fault-tolerance phases.
\end{abstract}

Index Terms-Fault-tolerance, cyber-physical systems, industry 4.0, fault detection, fault diagnosis, artificial intelligence.

\section{INTRODUCTION}

Fault-tolerant (FT) systems have been present in manufacturing environments since the last century [1], [2], addressing mainly the use of redundancy techniques and policies in equipment and devices to ensure system reliability. The adoption of redundancy techniques in areas with critical applications, such as aerospace [3] and power plants [4], are comprehensive from the associated risks due to its dangerous operating conditions. However, redundancy does not always result in the best solution in manufacturing automation systems. Also, project failures and technical limitations to implement FT in productive environments result in industries operating without FT systems, having to coexist with risks of failures and production shutdown, compromising the availability, performance, and quality of the production.

Technological advances associated with processing power, algorithm models, and communications techniques are growing at an impressive rate. The trend in FT systems is eliminating redundancy and enabling equipment to become self faulttolerant in a decentralized architecture. Therefore, according to the current scenario of the industrial sector, with privileged data crossing a cybernetic network and the boost in the number of equipment installed in the production line (e.g., robots and machines registered by the International Federation of Robotics (IFR) [5]), the number of episodes of failures tends to increase and can cause cascading effects on production, generating waste of time, resource and money. Understanding the functions, techniques, and challenges existing within the current FT systems represents an essential basis for extracting the most out of this approach in a prosperous factory of the future. In addition, Industry 4.0 [6] does not only require the simple development of systems based on disruptive technologies, but also that they exhibit robustness by applying these technologies based on a distributed, safe, reactive, agile, efficient, and collaborative architecture, i.e., with fault-tolerant characteristics in CPS environment.

Faced with this prosperous but complex industrial scenario, the scientific community is concentrating efforts to find solutions that are committed to the current industry demands, meaning avoiding failures, performance degradation, and productivity decrease [7]. In particular, research relating Industrial fault-tolerance policies in CPS results in excellent proposals based on IoT, AI, and cyber-security to ensure a continuous flow of flexible, dynamic, efficient, and safe production even in the occurrence of a disturbance during the production process. In other words, the system is capable of self-recovery from failures ensuring service.

Knowing the vital role that the FT will play in the new industrial CPS, this paper overviews the fault-tolerance and CPS concepts and performs a systematic literature review on applying FT in CPS to understand the current situation and on-going work in the field. For this purpose, the analysis of scientific papers retrieved from the Scopus repository in the last two decades was performed. Finally, the paper presents the research challenges linked to the development of FT in the context of industrial CPS.

The rest of this paper is organized as follows. Section II contextualizes the concepts and strengths regarding CPS and FT, and then contextualize the use of FT in CPS. Section III presents a systematic literature review related to the use of FT in the context of industrial CPS, providing the timeline evolution of the theme in the last two decades and highlighting the main topics and areas that underpin and prospect a 
potential FT advance found in the retrieved papers. Section IV discusses the main current research challenges considering the integration of FT in industrial CPS. Finally, the conclusions and future works are drawn in Section V.

\section{Cyber-Physical System AND Fault Tolerance: CONCEPTS AND STRENGTHS}

Considering the technological evolution and the demand of the manufacturing sector for scalable solutions that optimize the productive means, reduce costs, and maintain the quality, factories are adopting decentralized approaches in their production processes, following the Industry 4.0 philosophy [8], [9]. Therefore, to guarantee the dependability of operations, fault-tolerant industrial systems tend to migrate from centralized to distributed approaches, aligned with the emergence of industrial CPS. This chapter summarizes the concepts and strengths of CPS and FT and provides a contextualization of the contribution of integrating FT in industrial CPS.

\section{A. Overview of Fault Tolerance Concept}

Infallible systems are utopic because, in an industrial environment, the occurrence of an unexpected or unpredictable failure is a matter of time. A failure can be defined as an inadmissible deviation from a property or parameter of the system [10]. Consequently, a fault can generate a collective cascading effect and cause substantial financial loss, e.g., in an automobile manufacturing environment [11] or power supply [12], due to the malfunction of an actuator, loss of a sensor communication or disconnection of a system component. To avoid failures or mitigate their consequences, fault-tolerance techniques are being implemented to contribute to where the availability and dependability of a system are essential.

An FT system is one that can perform its function correctly and achieve its goals even in the face of an episode of failure. In other words, an FT system can mask and recover from a failure to continue operating correctly in the face of an anomaly [13]. Therefore, regardless of the reason of the failure, from software (e.g., high system complexity, specification failure, bugs, and implementation or malicious logic) or hardware (e.g., aging or wear, sizing error, electronic failures or mechanical failures), FT systems try to detect, diagnosis, isolate, and recover all functionalities of a system through the occurrence of an irregularity.

The development of FT techniques in industrial environments provides high availability for the execution of a specific industrial process. Therefore, the adoption of FT methods can avoid undesirable events within production systems. Considering that manufacturing systems are often deployed in dynamic environments where the operational conditions may change unpredictably, robust FT models can detect unforeseen failures in both hardware and software, avoiding undesirable collapses and enabling the process to be readjusted.

Consequently, the employment of FT techniques contributes to reducing unscheduled downtime, correct degradation performance, and increase product and employee safety in hazardous environments by detecting, isolating, identifying, and recovering the system as early as possible [14].

\section{B. Overview of Industrial CPS Concept}

Correlated to the 4th industrial revolution, the CPS concept has emerged to represent a complex integrated and networked system, exhibiting in-depth collaboration of computation, communication, and control technologies [15]. This requires the integration of embedded technologies in computational applications controlling and communicating with physical devices, where the interaction between cyber and physical elements occurs through a network, combining statistics, computational modeling and data [16]-[18].

CPS integrates several areas of technological knowledge into its structure to perform feedback control of physical and cybernetic entities, customarily distributed and heterogeneous, in real-time, in a safe, dynamic, collaborative, reactive, agile, and efficient way. In practice, CPS supports the digitalization of physical devices through the distributed collection of data using smart sensors, transmitted over a network, and processed in real-time. Indeed, this data is analyzed and processed, conditioning decision-making and actions, providing feedback control of the devices that integrate the complete system. In the industrial field, CPS can have a positive impact in the environments that have already digitized data from their production process, resulting in economic benefits, optimization of productive means, waste reduction, and avoiding production failures or deadlocks.

The implementation of such Industry 4.0 compliant solutions requires to combine the CPS approach with emergent technologies, e.g., IoT, AI, Big data and cloud computing, where each technology has an essential role in the current demands of digital factories. For example, IoT integrates communication across multiple devices and Machine to Machine (M2M), exchanging information between devices. Big Data enables the execution of techniques to obtain knowledge from a large volume of data, e.g., using AI to support the extraction of patterns for autonomous decision making. Cloud computing enables the massive storage of data from the digitalization processes, as well as high processing and computational analysis power.

The adoption of CPS systems corresponds to the transition from industrial systems based on traditional patterns, centralized with isolated components and without communication to a decentralized and self-organized system that provides agility, responsiveness, and adaptation to changing conditions and decision-making, enabling energy efficiency and effective cost [16], [19]. In this context, CPS foster the implementation of large-scale systems in the most diverse "smart" areas, such as smart manufacturing, smart warehouse, smart grids, smart buildings or smart farms.

\section{Migrating Fault Tolerance for the Industrial CPS Context}

In this context, emergent technologies like IoT, wireless sensor networks, cloud computing, AI, autonomous agents, are being increasingly integrated into modern industry [20], 
[21], contributing to improving current systems [22]. As each equipment becomes an intelligent, precise, efficient, and autonomous agent, it may have the capacity for self-diagnosis of failures, checking if an identified failure is local or global through the interaction with other distributed agents. The application of FT methods in CPS contributes to an optimized production process, leaving behind the traditional failure and fix maintenance methodology (FAF) replaced by the predict and prevent (PAP) approach [11], [23].

However, there are limitations regarding the use of FT in CPS environments, mainly because decentralization and distribution impose crucial challenges for the implementation of FT models in industrial systems. It is essential to know how to use the massive amount of available data to identify, diagnose, and predict failures, not only the monitorable faults (superficial or apparent). To benefit from this volume and availability of data to conduct profound real-time prevention of equipment failures, it is important to take corrective actions to recover the system without impacting the industrial process. Following this work, a systematic review of using FT in CPS will be performed, as well as the challenges that emerge in this topic will be highlighted.

\section{Systematic Literature ReVieW}

As previously described, an emergent research field appears in the Industry 4.0 context by combining the intrinsic complementary FT and CPS concepts. Aiming to survey and analyze the current panorama of using FT systems in the CPS context, understanding the horizons and assessing the benefits of these techniques within industrial scenarios, and supporting the extraction of conclusions of research directions, a systematic literature review was conducted. The systematic review considered the data retrieved from the Scopus repository regarding digital scientific publications, resulting in a wide range of work in a fast, efficient, and much larger depth when compared to more traditional manual review methods [24]. With this effort, it is possible to analyze a sequence of placements and terms, particularly crucial for the development and basis for appropriate research.

The methodology consists of searching specific terms on the "Title", "Abstract" and "Key-words" of scientific papers available in the Scopus repository. The search focused on the appearance of the combination of CPS and FT terms in the Scopus repository, using a query, illustrated in Fig. 1), that comprises 28 query combinations.

The implemented query retried 341 papers, which yearly evolution is shown in Fig. 2, being possible to observe that the academic community started to address this area in 2007 in a very isolated way. After launching the Industry 4.0 initiative, in 2013, an expressive increase of papers related to the use of FT in CPS was noticed. This means that emergent technologies, e.g., IoT, cloud computing, and AI, entered the industrial scenario prospecting a new procedure for FT systems. These tools complement the redundancy of the equipment, inserting agents capable of identifying failures and performing actions based on the volume of information available. For this new

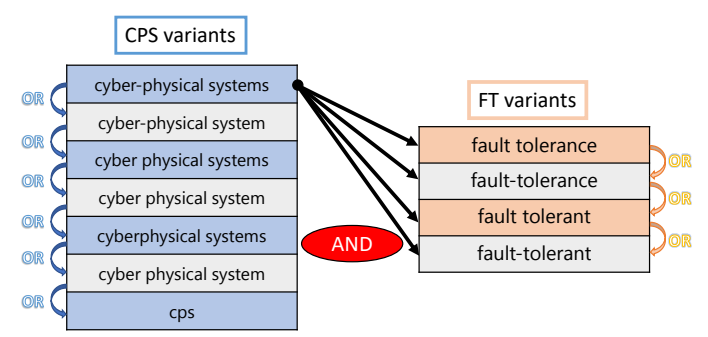

Fig. 1. String query to retrieve data related to papers containing FT and CPS from the Scopus repository.

decade, the factories of the future will demand technologies that integrate FT within the industrial CPS to increase the availability of the production process, then a positive evolution in this research topic is expected.

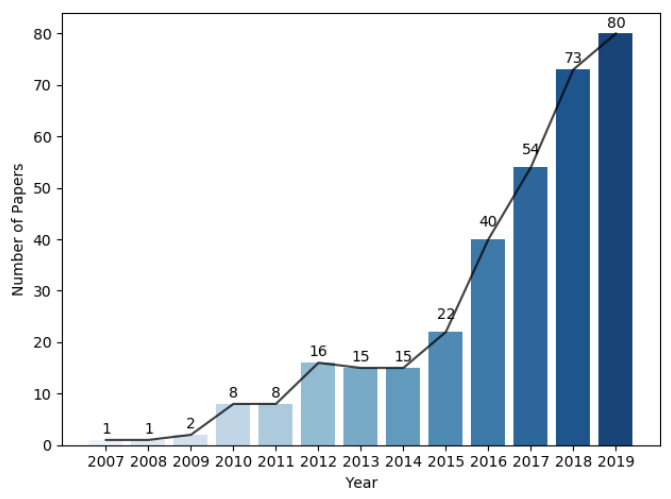

Fig. 2. Evolution of the number of papers published per year.

Considering the retrieved data, a search for combinations of $\mathrm{N}$-grams, namely bigrams and trigrams, was also performed, aiming to catalog particular topics and technologies related to the application of FT in CPS environments that the academic community has often been addressing.

Fig. 3 shows the result for searching bigrams, which are ranked by raw frequency. As expected, "cyberphysical system" was the most relevant term, followed by fault-related terms, e.g., "fault detection", "fault-tolerance", and "fault diagnosis". The appearance of "internet thing" and "sensor network" highlights the importance of ICT technologies in linking FT and CPS. The manifestation of "smart grid" and "power systems" in the research indicates that the energy production domain has a special interest in distributed FT systems to ensure robust solutions. Sensor-related areas also appear frequently in the search, e.g., "sensor network", "wireless sensor", " sensor data" and "sensor actuator", assuming crucial relevance in the data collection. Finally, terms such as "neural network", "machine learning" and "deep learning" appear in the search space, showing the potential of AI tools for data analysis, decision making, and failure event prediction.

A similar understanding can be observed in the most frequent trigrams presented in the retrieved data set (see Fig. 4). A reinforcement of the focus on "cyber physical system" and 


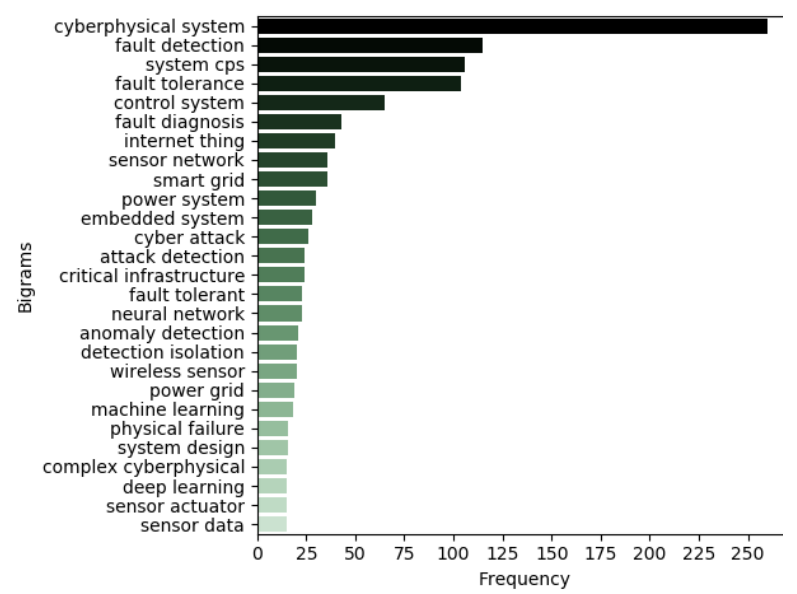

Fig. 3. Bigrams ranked by raw frequency of appearance.

the variation in topics related to failure situations, e. g., "fault detection isolation" and "fault detection diagnosis" are noted.

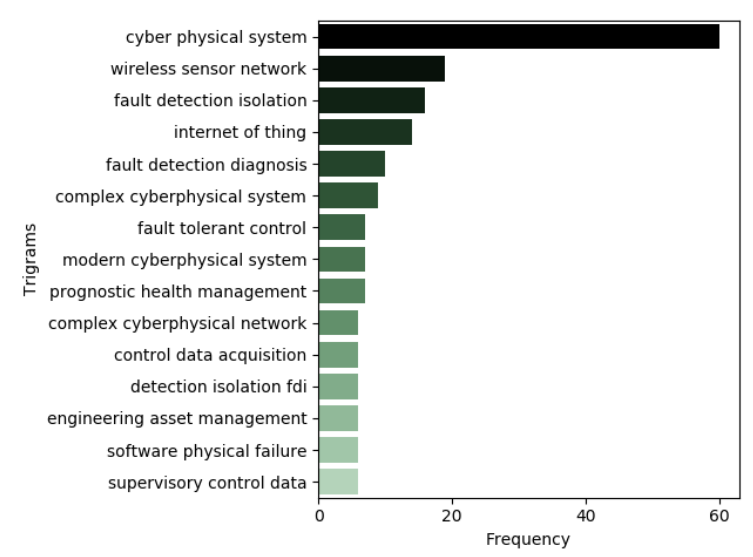

Fig. 4. Trigrams ranked by raw frequency of appearance.

In addition to the $\mathrm{N}$-gram analysis summarized above, a bibliographic coupling analysis was also conducted to analyze the research community in this field (see Fig. 5). This analysis corresponds to the number of common citations (either from within or apart from the set of papers analyzed), correlating the papers retrieved from the Scopus query.

The analysis of these results shows that during the last decade, fruitful results have been reported on using FT in CPS to improve diagnosis, isolation, and fault recovery, enhancing production processes. FT has been following the new industrial-technological scenario and is incorporating new technologies to benefit and avoid production failures. However, this research network still is in an initial development stage.

\section{Current Research Challenges in FAULT-TOLERANT CYBER-PHYSICAL SYSTEMS}

As previously presented, the migration of traditional FT systems into CPS environments have a high potential of applicability within the industrial sector. However, due to the rapid

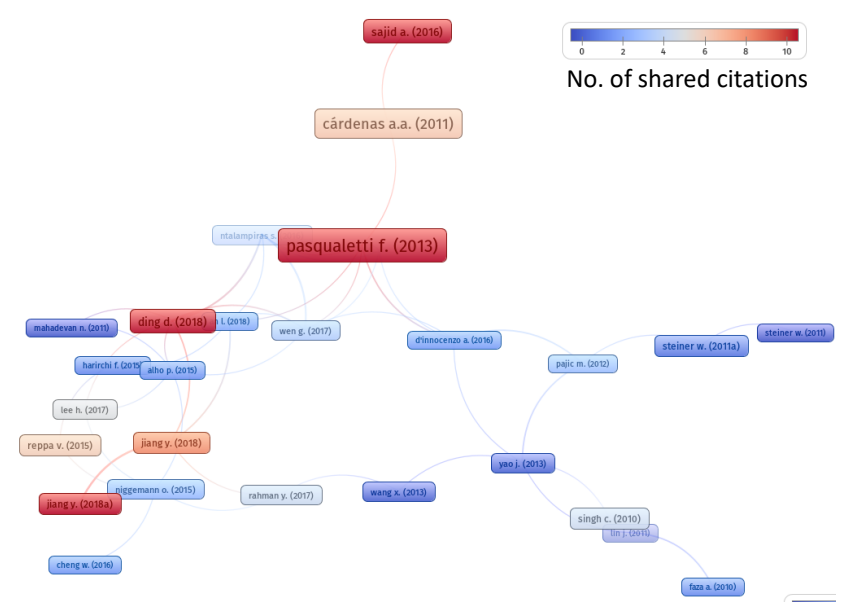

Fig. 5. Bibliographic coupling analysis based on references that are shared among the papers.

progress of technologies and manufacturing processes, there are several challenges for this sector to be entirely benefited by using these emergent technologies that will support distributed and decentralized solutions. This section will briefly discuss some research challenges, clustered in four groups (see Fig. 6): data collection, data analysis, security and privacy, and system restore.

\section{A. Data Collection}

With the continuous exponential increase of connected devices from different vendors in industrial environments, data collection becomes increasingly complex and challenging. The collection of data aiming to extract valuable information about failures [11] is usually constrained by interoperability problems due to the need to collect data from a large number of heterogeneous sources and devices. Therefore, the challenge is related to develop robust and interoperable systems with welldefined data models, e.g., AutomationML, and compatible communication protocols, e.g., OPC-UA.

Smart sensors play a crucial role in collecting valuable, accurate, and reliable data in an industrial environment. For this purpose, they have embodied local processing power to pre-process local measurements and improve the output data. In this sense, fostering the application of IoT nodes with edge computing technologies is a promising aspect of the smart sensors field. Another challenge is related to selfdiagnosis faults in a distributed manner using the IoT and edge computing technologies. For this purpose, these distributed agents should monitor their internal parameters and identify deviations that correspond to real faults early.

Aiming to develop distributed FT systems, to is crucial to implement data synchronization (avoiding missing or copy of data) and fusion, since these systems are based on the collection of a massive amount of data coming from different heterogeneous sources.

Finally, the detection, diagnosis, and consequently, the treatment of failures suffer from delays due to the time associated 

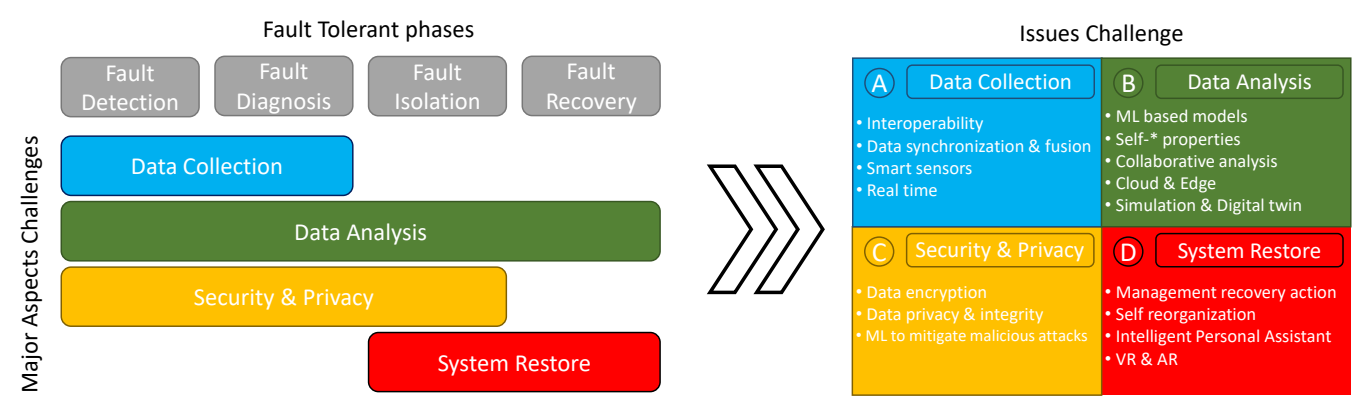

Fig. 6. Challenges aspects to implement fault-tolerance in industrial CPS environments.

with data collection and processing. It is necessary to collect and transmit data in real-time to improve the robustness of FT in CPS systems. Advances in 5G technology within the industrial sector can contribute to this aspect with a boost in the systems' responsiveness [25].

\section{B. Data Analysis}

The production sector is incorporating the benefits of using AI [26]. However, the application of FT in CPS environments still faces the barriers of developing generic, distributed, and scalable models to all fault-tolerant phases, i.e., detection, diagnosis, isolation, and recovery, which allows to simplify and reduce the deployment time. In the near future, the challenge of applying ML-based models in FT applications tends to enable autonomous systems with self-* properties. For example, self-configuration behaviors will adapt the system parameters dynamically to face the condition changes. Selfhealing will allow discovery and diagnosis faults in the factory, and Self-protecting will allow to anticipate, detect, and protect the manufacturing systems against faults.

Being a distributed system, where each agent can collect and analyze data, a significant challenge that arises is related to the collaborative analysis. In particular, issues regarding how a collective analysis is performed based on the contributions of several entities will be crucial, allowing the decision-making dependency from a central node to be removed. Thus, each agent/device can detect the occurrence of failures by itself, or even inform other agents of the existence of failures, culminating in the collaborative analysis.

In this context, the distribution and balancing of intelligence among Cloud and Edge computational layers assume a critical aspect in implementing this new FT approach. In particular, the cloud can be used to process a huge amount of aggregated data and train the AI models, that will be later deployed in the individual nodes hosted at edge computational layer. This allows to predict failures or states for recovery to operate in the presence of failures in an earlier stage at edge level.

Simulation and digital twin are other essential technologies in FT domains. The challenge is not to create virtual prototypes of the scenarios and simulate failures, but instead to represent the production system to determine the desired state and compare it with the real conditions to anticipate faultserrors or deadlocks might occur over time.

\section{Security and Privacy}

Security and privacy issues within the CPS also contain challenges related to the development of distributed FT systems, namely in terms of security systems to ensure the safety of humans, the environment, and machines against the consequences of malicious attacks. Regularly, many new approaches seeking new metrics of malicious attacks are developed, and consequently, security measures must face these attacks without compromising the data that traffic in the industrial network.

Many of the protocols used were not designed given the high volume of data being transmitted by the different layers of the industrial sector traveling over the internet. All devices need to use encryption of stored and send data, and secure approaches against malicious attacks, to ensure that no unauthorized entity has access to private data [27]. In this sense, new approaches to encrypt the data that transit through the industry are required.

The adoption of block-chain technology to maintain data privacy and integrity allows increasing the security by building key structures to encrypt confidential information and prevent malicious entities from tampering with information. Another critical challenge concerns the implementation and development of ML-based mechanisms to mitigate malicious attacks and prevent exposure.

\section{System Restore}

Systems restore techniques that culminated in failures represent an enormous relevance within distributed FT since as long as the system is not reconstituted, countless losses can occur. A significant research challenge, at the software level, refers to the system itself being able to reconfigure and recalibrate, to overcome a failure event with the least possible loss, using AI-based management recovery action policies.

Currently, it is not always possible to recover automatically from system failures. Hardware failures usually require replacement or maintenance, and human involvement in such tasks is still necessary. However, technicians can be assisted with intelligent personal assistant (IPA) systems contributing to perform the system restore in a faster and efficient manner. Virtual reality (VR) and augmented reality (AR) can also provide useful real-time information about the system status, corrective measures, and recommendations to instruct the best procedures during these interventions in case of unexpected 
failures. The challenges associated with IPA, VR, and AR technologies are related to the development of systems that have a complete and integrated virtual model of the production process that is capable of predicting all possible failures and intuitively supporting the interventions, besides the ergonomic and adaptation of the operator to the use these technologies.

\section{CONCLUSIONS}

With the advent of the 4th industrial revolution, modern systems have a more complex control, which brings critical challenges to systems safety and reliability. In this context, fault-tolerant systems need to be improved to operate in conjunction with disruptive ICT technologies within a distributed and decentralized environment, promoting methods of maintaining operation in failures' episodes.

FT systems attend this notorious expectation of obtaining a system capable of detect, diagnose, isolate, and recover from failures, as shown by the systematic review of the literature conducted in this work. The analysis of the bigrams and trigrams highlighted some important topics in deploying FT in CPS, namely IoT, sensor network, smart grid, and cyber-attack, as well as other topics related to distributed and decentralized architectures, representing the importance of embodying these emergent technologies with the traditional and reliable methods. This work also discusses some relevant challenges when developing distributed and decentralized FT systems, namely data collection, data analysis, security and privacy, and finally, system restoration, cross-matching with the fault-tolerant phases.

As future work, some of these challenges will be deeply researched, particularly developing fault-tolerant systems for a smart cyber-physical warehouse system.

\section{ACKNOWLEDGMENT}

This work has been supported by FCT - Fundação para a Ciência e Tecnologia within the Project Scope: UIDB/05757/2020. This work has been partially supported by the European Regional Development Fund (ERDF) through the Interreg Spain-Portugal V-A Program (POCTEP) under grant 0677_DISRUPTIVE_2_E (Intensifying the activity of Digital Innovation Hubs within the PocTep region to boost the development of disruptive and last generation ICTs through cross-border cooperation).

\section{REFERENCES}

[1] N. A. Duffie, R. Chitturi, and J.-I. Mou, "Fault-tolerant heterarchical control of heterogeneous manufacturing system entities," Journal of Manufacturing Systems, vol. 7, no. 4, pp. 315-328, 1988.

[2] P. R. Chintamaneni, P. Jalote, Y.-B. Shieh, and S. K. Tripathi, "On fault tolerance in manufacturing systems.," tech. rep., Maryland Univ College Park Inst for Advanced Computer Studies, 1987.

[3] J. W. Bennett, G. J. Atkinson, B. C. Mecrow, and D. J. Atkinson, "Fault-tolerant design considerations and control strategies for aerospace drives," IEEE Transactions on Industrial Electronics, vol. 59, no. 5, pp. 2049-2058, 2011.

[4] N. Ertugrul, W. Soong, G. Dostal, and D. Saxon, "Fault tolerant motor drive system with redundancy for critical applications," in Proceedings of the IEEE 33rd Annual IEEE Power Electronics Specialists Conference., vol. 3, pp. 1457-1462, IEEE, 2002.
[5] I. F. of Robotics, "Executive summary world robotics 2019 industrial robots."

[6] H. Kagermann, W. Wahlster, and J. Helbig, "Securing the future of german manufacturing industry: Recommendations for implementing the strategic initiative industrie 4.0," Final report of the Industrie, vol. 4, no. $0,2013$.

[7] Z. Gao, C. Cecati, and S. X. Ding, "A survey of fault diagnosis and fault-tolerant techniques-part i: Fault diagnosis with model-based and signal-based approaches," IEEE Transactions on Industrial Electronics, vol. 62 , no. 6 , pp. 3757-3767, 2015.

[8] M. Brettel, N. Friederichsen, M. Keller, and M. Rosenberg, "How virtualization, decentralization and network building change the manufacturing landscape: An industry 4.0 perspective," Int. Jour. of Mechanical, Industrial Science and Engineering, vol. 8, no. 1, pp. 37-44, 2014.

[9] Y. Lu, "Industry 4.0: A survey on technologies, applications and open research issues," Journal of Industrial Information Integration, vol. 6, pp. 1-10, 2017.

[10] D. van Schrick, "Remarks on terminology in the field of supervision, fault detection and diagnosis," IFAC Proceedings Volumes, vol. 30, no. 18, pp. 959-964, 1997.

[11] Y. Xu, Y. Sun, J. Wan, X. Liu, and Z. Song, "Industrial big data for fault diagnosis: Taxonomy, review, and applications," IEEE Access, vol. 5, pp. 17368-17380, 2017.

[12] B. Mirafzal, "Survey of fault-tolerance techniques for three-phase voltage source inverters," IEEE Transactions on Industrial Electronics, vol. 61, no. 10, pp. 5192-5202, 2014.

[13] A. S. Tanenbaum and M. Van Steen, Distributed systems: principles and paradigms. Prentice-Hall, 2007.

[14] H. Lee, "Framework and development of fault detection classification using iot device and cloud environment," Journal of Manufacturing Systems, vol. 43, pp. 257-270, 2017.

[15] Y. Liu, Y. Peng, B. Wang, S. Yao, and Z. Liu, "Review on cyber-physical systems," IEEE/CAA Jour. of Autom. Sinica, vol. 4, pp. 27-40, 2017.

[16] J. Barbosa, P. Leitão, D. Trentesaux, A. W. Colombo, and S. Karnouskos, "Cross benefits from cyber-physical systems and intelligent products for future smart industries," in Proc. of the IEEE 14th Int'l Conference on Industrial Informatics (INDIN), pp. 504-509, IEEE, 2016.

[17] A. W. Colombo, T. Bangemann, S. Karnouskos, J. Delsing, P. Stluka, R. Harrison, , et al., "Industrial cloud-based cyber-physical systems," The IMC-AESOP Approach, vol. 22, pp. 4-5, 2014.

[18] P. Leitão, A. W. Colombo, and S. Karnouskos, "Industrial automation based on cyber-physical systems technologies: Prototype implementations and challenges," Computers in Industry, vol. 81, pp. 11-25, 2016.

[19] S. Karnouskos, A. W. Colombo, T. Bangemann, K. Manninen, R. Camp, M. Tilly, P. Stluka, F. Jammes, J. Delsing, and J. Eliasson, "A soa-based architecture for empowering future collaborative cloud-based industrial automation," in Proc. of the IECON 2012-38th Annual Conference on IEEE Industrial Electronics Society, pp. 5766-5772, 2012.

[20] J. Wan, S. Tang, Z. Shu, D. Li, S. Wang, M. Imran, and A. V. Vasilakos, "Software-defined industrial internet of things in the context of industry 4.0," IEEE Sensors Journal, vol. 16, no. 20, pp. 7373-7380, 2016.

[21] L. Kong, D. Zhang, Z. He, Q. Xiang, J. Wan, and M. Tao, "Embracing big data with compressive sensing: A green approach in industrial wireless networks," IEEE Communications Magazine, vol. 54, no. 10, pp. 53-59, 2016.

[22] P. Leitao, S. Karnouskos, L. Ribeiro, J. Lee, T. Strasser, and A. W. Colombo, "Smart agents in industrial cyber-physical systems," Proceedings of the IEEE, vol. 104, no. 5, pp. 1086-1101, 2016.

[23] T. Sutharssan, S. Stoyanov, C. Bailey, and C. Yin, "Prognostic and health management for engineering systems: a review of the data-driven approach and algorithms," The Journal of Engineering, vol. 2015, no. 7, pp. 215-222, 2015.

[24] H. Yasin, M. M. Yasin, and F. M. Yasin, "Automated multiple related documents summarization via jaccard's coefficient," International Journal of Computer Applications, vol. 13, no. 3, 2011.

[25] S. Li, L. Da Xu, and S. Zhao, "5g internet of things: A survey," Journal of Industrial Information Integration, vol. 10, pp. 1-9, 2018.

[26] J. Lee, H. Davari, J. Singh, and V. Pandhare, "Industrial artificial intelligence for industry 4.0-based manufacturing systems," Manufacturing Letters, vol. 18, pp. 20-23, 2018.

[27] E. Sisinni, A. Saifullah, S. Han, U. Jennehag, and M. Gidlund, "Industrial internet of things: Challenges, opportunities, and directions," IEEE Transactions on Industrial Informatics, vol. 14, no. 11, pp. 4724-4734, 2018. 